\title{
The Evolving Collaboration - AMazed! The A-Maze artists investigate immersive technology to create imagination and artifice
}

\author{
Maureen Kendal \\ Artist / Director \\ DreamStudio.io \\ maureen@dreamstudio.io \\ Chen Mei-Tsen \\ Artist \\ www.chenmeitsen.com \\ meitsen668@gmail.com
}

\author{
Fion Gunn \\ Artist \\ http://www.fiongunn.org \\ fiongunn@live.co.uk \\ Terri M. Broughton \\ 3D artist \\ https://terribanimation.weebly.com \\ fiongunn@live.co.uk
}

\author{
Nazia Parvez \\ Artist / Designer \\ Instagram: @nzp_skechbook \\ nzparvez@gmail.com
}

Cleon Grant

Software Developer

cleonkg@gmail.com

This paper examines the construction of an artificial edifice, a navigational architecture, which connects A-Maze Artists' world and development of toolkits for artists to enhance their working practice. The cross-generational, culturally diverse A-Maze artists use a trans-disciplinary approach, mutual interviewing techniques, insights from online meetings, continuous evaluation, iterative design-decisions, critical challenges and questioning. A-Maze artists investigate their working practices, share visual imagery, symbolic language, identifying differences and shared interests. Within these conversations, the artists have been reconsidering, shifting ground, transforming their practice and vision in response to their co-collaborators. A-Maze have reached out to artists from various other regions internationally. Previous research by A-Maze artists explored virtual technologies by artist/practitioners, identifying issues around ideas of 'natural', 'random' and 'uncanny'; access to technologies; ethical frameworks and digital regulation. Today A-Maze includes artists have diverse skill-sets covering areas such as architectural/service user design; 3D character animation; 3D development; cartography; curatorial, public engagement animation and cyber/virtual eco-landscapes. They build on their histories of migration and lockdown, using virtual tools and/or visualisation to realise these interconnected immersive worlds to present: 'Boundless: Worlds in Flux'. Within these worlds, participants visit interconnected immersive multiverses of visual imagery, sound and text. From our age of the Anthropocene, AMaze imagines Virtual Worlds, transposing ideas from landscape to virtual-scape. Exploring narrative navigation through the use of maze, game, cycle, metaphor and avatars. Embracing cocreation in a meaningful way through imaginative, inventive curation.

VR. Navigation. Virtual worlds. Storytelling. Virtual cultural heritage. 2D and 3D imaging.

\section{INTRODUCTION}

A-Maze Artists Group comprises six artists from different backgrounds, nationalities and generations who are developing an interactive, immersive virtual reality experience. Thematically, A-Maze is focused on the idea of "flux" as a key to understanding the complexity of our world. This includes the fluctuations of marine life, plant growth and climate change, human migrations and collective memory. The key themes are interconnected systematically throughout: ecology, trans-disciplinary practices, collaboration, interconnectedness, connectivity, community, sitespecific interventions, audience-participant-user engagement, metaphor, symbolism, multiple perspectives, transformation, metamorphosis, inclusivity, combating digital exclusion, empathy and ethics.

Key features are:

1. Methods to investigate the artists' and the users' design requirements and technical specifications including mutual interviews, regular team meetings, artists' statements.

2. Individual environments where artists are autonomous with a common hub through which viewers enter, all areas in the VR space are interconnected and form a multiverse. 
3. Creation of a common navigational framework, which connects the unique artists' spaces, underpins the design, development and implementation of features and functions.

4. Design of a participants' space for cocreation.

Inspired by and building on Fion Gunn's work for Tate Exchange Liverpool (Gunn, 2019), the 'Odyssey' project, this Virtual Reality project 'Boundless' takes the users for a journey conceptually between the macrocosm and the microcosm within this interconnected vibrant multiverse.

Building on her Odyssey project and in response to the CoVid-19 Lockdown, Fion Gunn, 2020, invited fellow artists to join her for the development of Artists' Virtual Worlds. This collaborative research, practice and exhibition is now being built on in this project. Research includes virtual avatar worlds, VR tools and globally connected participatory workshops (Hudson \& Kendal 2011; Kendal et al. 2019; Gunn 2019; Gunn et al. 2020).

Cleon Grant 2021 reflects: "As life has changed over time for many, our world has become more connected and yet disconnected. CoVid allows new methods of expression and connection through distance. The worlds in flux represent how our real world has begun to collide with our digital worlds."

Mei-Tsen Chen 2021, states: "CoVid-19 completely changes our interactions with other people which push us into a frenetic digital Web. As the world is busy in search of better connectivity and a more efficient way to save Big Data. As an artist I am trying to understand the meaning of this pandemic, which we experience now. I am sharing my questioning with the other artists of the A-Maze group, and to know how our universes can be interwoven together despite our difference in age, culture, geography and personal stories. Observing Nature to realise how we are intimately connected with our environment, to study the mutual relationships between living creatures and their biological, social, and environmental surroundings. Using digital means to translate this feeling of displacement in flux, in a fragmented, uncertain and random world."

The priority is how the user will experience the project, its quality, its accessibility and inclusivity. The focus groups are reflective of a wide range of age, ethnicity, cultural and economic backgrounds. The feedback of these participants drives the iterative prototype development. As CoVid lockdown and physical social distancing starts to be relaxed, the project will expand to encompass tangible, physical and performative aspects. Currently funding is secured for the Minimum Viable Product (MVP) development phase, led by artist Fion Gunn (Arts Council England 2021).

\section{METHOD}

\subsection{Methodology and process}

Kendal and Gunn discussed potential collaboration in early April 2020, after which Gunn identified a number of artists in her network and invited them to join. From the outset we have been committed to diversity, cross-generational participation, egalitarianism and being women-led. From then on weekly and often bi-weekly online meetings have been held to discuss ideas, methodologies and to formulate the parameters of the project. Once a number of key themes were established, Gunn created a first draft of the VR experience in Tilt Brush in collaboration with each artist.

The curatorial approach was agreed early on artists would be autonomous in their own environments, there would be a common hub through which viewers would enter and all areas in the VR space would be interconnected, in effect we wanted to create a multiverse. We share resources and information. We are flexible, responsive to circumstances and evaluate our creation critically on an ongoing basis.

Through a series of mutual interviews, one to one and across the team, ideas for the navigational architecture, features and functions were generated. These ideas draw inspiration from open ongoing discussions around each artists' philosophy and practices, passionate inspiration, impact of their artwork and the artists' relationships with their audiences. Links to the Artists' Statements and extracts of interviews are included to illustrate the conversational flow. Interview material was analysed to propose a common framework of navigational pathways, features and functions.

\subsection{Artists' design investigations}

A-Maze artists' are using traditional practices, digital media, and virtual tools to realise their Artists' spaces through interconnected immersive worlds. A-Maze is designing and developing a minimal viable product (MVP) for 'Boundless: Worlds in Flux'. A-Maze is approaching this virtual world development differently from the traditional computer game world. The viewer is invited not just to play but also to become a witness and to participate in transformative activities and cocreate. 

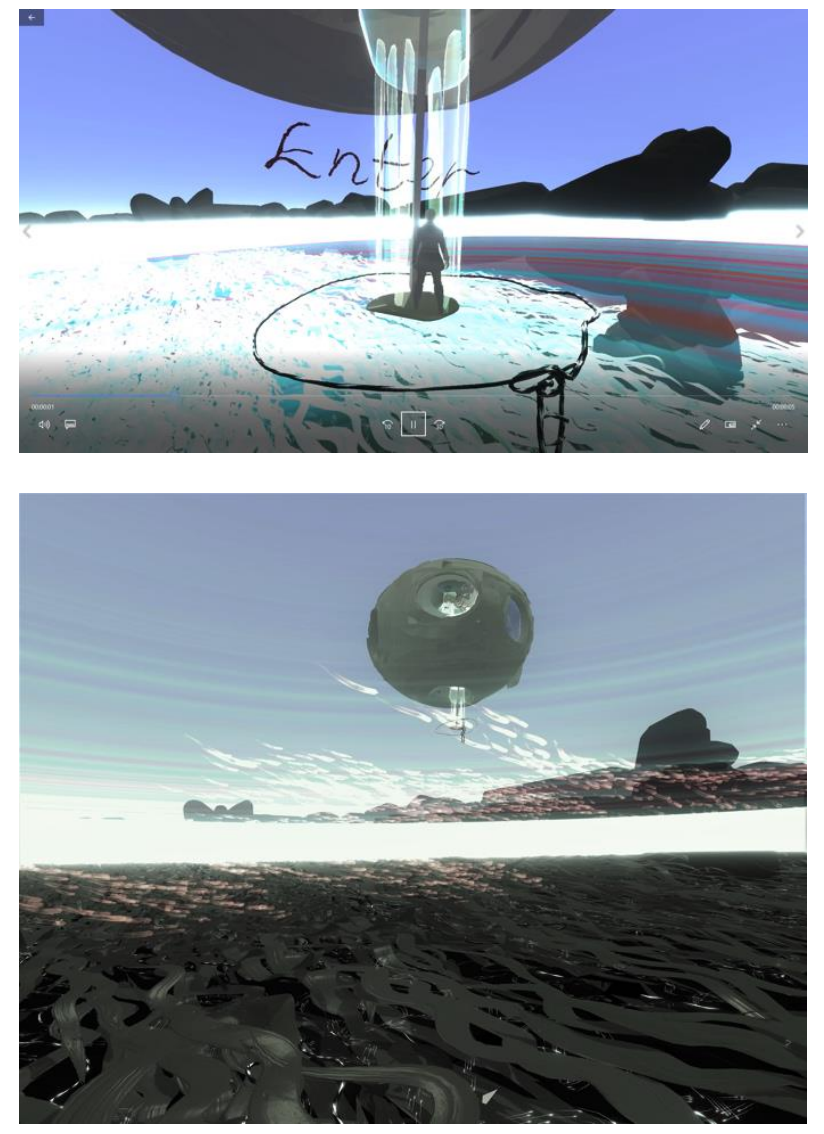

Figure 1: Visual evidence.

The artists have been experimenting with:

- How the immersive tools transform their artistic practices

- How the artists, by means of their artistic practice, have responded to the CoVid pandemic

- How collaboration with the other A-Maze artists has influenced the development of their individual practices

- How can the design of navigation and interface enable the users (audience/ participants) to engage fully in this virtual immersive world

- What strategies can reach out to appeal and bring in audiences who do not use any gaming or immersive platforms

The artists' discussions centre around their creative practices, philosophy, ethics and the prioritisation of empathy. They probe each others' concepts, focus, lived experience and aesthetic sensibility. They investigate how these drivers impact their design-decisions for the functions and features required in this virtual world, these will determine its navigation, design and interactivity.

\subsection{Conceptual concerns}

The artists, working in collaboration, identified conceptual areas to investigate:

1. Metaphors and concepts that translate themselves into enablers/features and functions that afford the user experience within the virtual world. (Gunn 2021).

2. The design of the space by the artists and the creative discussion between the artists - generate ideas of the user's engagement and functionality.

3. Conceptual thematic and functional-come together in terms of ideas and functions. The artists are creating a personal space and experience however the collective dialogue points towards a common language of concepts, despite cultural and linguistic differences.

4. Notions of miniaturisation, the manipulation of scale, a mini theatre, microcosm, digital dolls' house and online gaming, enable the user to act out cathartically real life every day emotions and conflicts within an immersive yet safe space.

\section{NAVIGATIONAL ARCHITECTURE}

\subsection{Overall system and map of the virtual world}

The navigational architecture provides a common framework from which to explore each individual environment and builds on nautical themes and marine life. The viewer has various options for navigation; one of these includes diving underwater and approaching a spherical hub through shoals of fish and emerging underneath the structure. The viewer then travels upward into a sphere with portals opening onto different worlds.

Multiple pathways allow viewers to travel between the artists' worlds or return to the central space (Gunn 2021). An example of collaborating on the design decisions for how navigational architecture can connect the A-Maze worlds led to a discussion around the application of physical laws and gravity in a virtual space. One option proposed that the transportation portal move and function as if in the real world, with the user having to follow a linear sequence of actions to move from an underwater environment to the land above. This was rejected in favour of an open horizontal user flow across all portals, in order to convey the significance of analogy and metaphorical thinking (Chen and Kendal 2021). 
The Evolving Collaboration - AMazed!

Maureen Kendal et al.
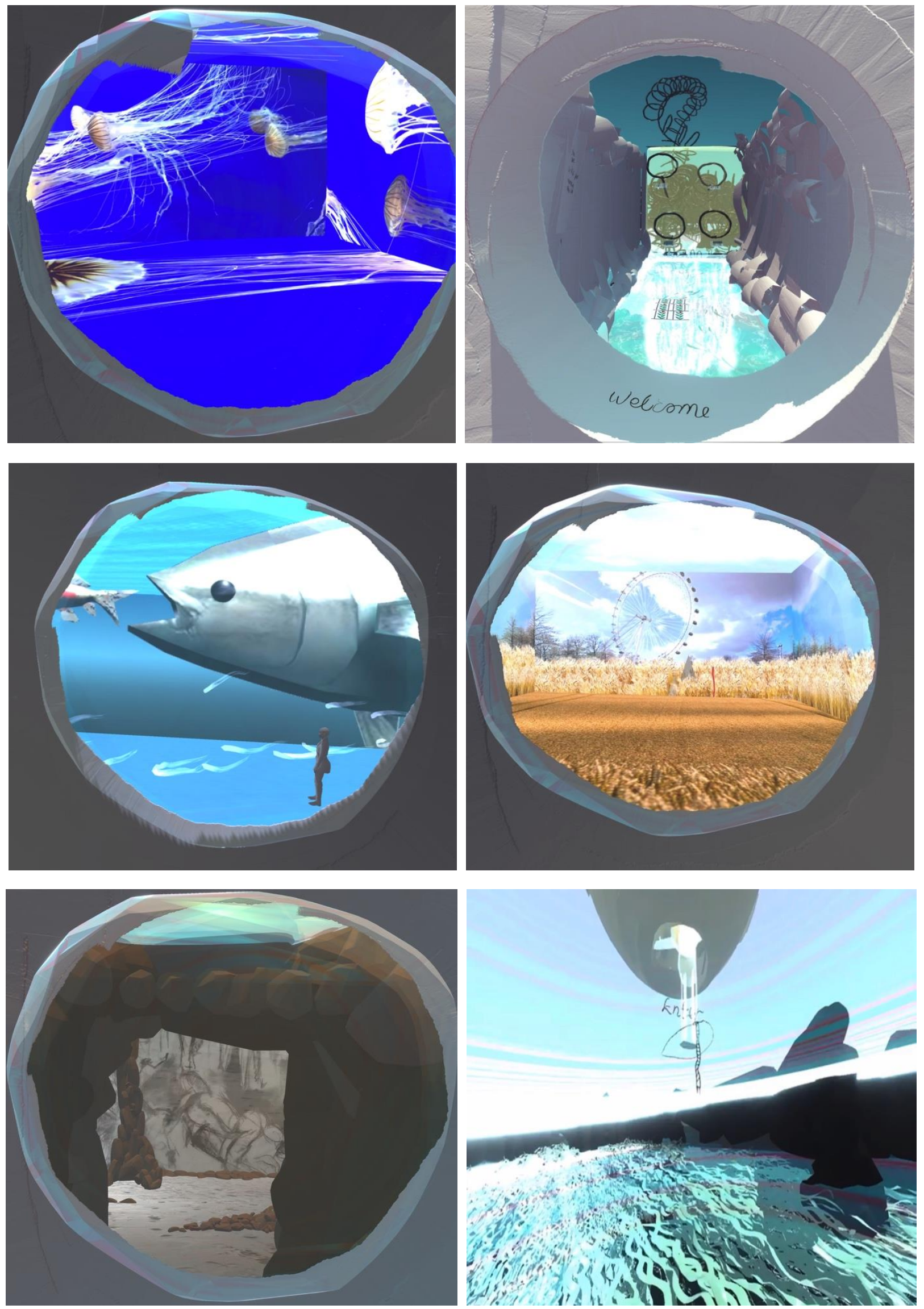

Figure 2: Visual evidence. 


\subsection{Common framework for an architectural and navigational requirements}

Within each of these spaces, all artists are designing navigational functions, key triggers and pathways for the users, to include signs and signposts, navigational controls, audio and transportation portals for between artists' spaces.

Within Unity, pre-sets from Unity asset store or created customisable coded behaviours are used (Unity Technologies, 2021). Example One: transportation portals are set up to move the user/camera from (position 1 set of coordinates) to (position 2 set of coordinates). This script will be programmed into an Object or Event. Objects are specific locations e.g. a tunnel or an Event-Trigger loaded onto a button. Example Two: embedded views will build up into a database to collect 3D and tracking information, to monitor when a user is interacting with the virtual space. The data pattern collection will output to a dashboard or display within a physical gallery space and online. This data collection around the user journey, duration and locations is used as a measure of successful engagement.

Following design requirements, technical development and iterative testing will inform the development of the MVP. System requirements are available on www.worldsinflux.com (Grant, 2021).

\subsection{Navigational key-points}

- Straplines

- Guides - animated creatures

- Prompts - 'Follow that Fish' - pulsating or Gesture, movement mechanics

- User point of view

- Live-links to site specific location: future potential partnership

- Embed Viewpoints

- User pathways, signs and signposts

- Navigation controls

- Audio

- Transportation Portals

- Assets/ Art-works/ Avatars: collectibles; Character; Selfies

- Multi-verse of narratives - environments

- Outcomes include takeaways, measures of engagement, collection and display of metrics.

\subsection{Rules of play}

The rules of play in our multiverse are being designed and developed. There is a common structure for common areas. These connect and make sense with the individual artists' worlds structures - the rules of play are unexpected and surprising so as to challenge and stimulate the viewer. The rules of play enable the user to experiment through the pathways to discover a specific sound track, they can pick up part of a coil, a fish. There are photo opportunities for people to take an image of the girl avatar and upload their own images - sitting on the carpet and playing with scale. People love playing with the Qlone app in Tate Liverpool (Gunn 2021).

Features include:

A Hub, which gives users access to each artist's world/space/exhibition. Users navigate their way through the interrelated and interconnected artists' worlds via portals embedded in each Artist's world offering up to 36 Transportation Portals.

Interactive Hotspots with easy access and in any sequence, providing users with an open-ended experience. Hotspots can offer narrated stories, video clips, soundscapes and info-grams.

Participant's space/visitor's world 'Your Space' allocates users a co-creative role, enabling them to create their own collage-assemblage with media uploads and collected assets and 'collectibles' from their Boundless journey. Asset types may be movies, images, sound and writing. 'Your Space' is a generative space, growing in size and complexity over time.

Pathways with triggers and trails are experienced through a gamer first person view, subsequently developed as Augmented Reality AR applications, with markers/QR codes, pop-ups and sensors positioned in physical reality as triggers. Built-in timing will facilitate health and safety requirements for distancing. Sensors will trigger projections, sound etc. as visitors move through the physical space. Pop-ups will be excerpts from the Boundless environments, which reference the host venue's own collections. The trails can be laid out in ways that lead visitors to discover collections they may not have thought about before, they can be used as part of the overall curatorial vision for the venue and A-Maze anticipates collaborating closely with any venue's curatorial team.

The user experience is expansive, diverse and immersive, a multiverse which people want to visit repeatedly because of its enriching experiences, multiple pathways and opportunities to co-create, collaborate and join the 'Boundless' conversation. Outputs offer an online destination for schools, communities, special interest groups or friends to meet up within this online hub, which is linked to a cultural location, gallery, museum or specific collection.

In essence, the rules of play, its features and functions will allow the user to hunt and gather collectibles, encouraging users to collect, frame 
and re-assemble, composite traces, memories and remnants. This experience of virtual immersive can offer the user/audience - 'head space' to encourage their imagination. Using juxtaposition of significant objects, the user makes sense of their choices or design decisions, creating a surrealistic quality. Gunn 2021 explains: "We like certain things, but why? Gunn recalls that she likes " transparent things with spots on, absolutely magical patterns. These are the sensations (she recalls) from childhood." Gunn reflects: "We underestimate our memories, our sensory states; to discover the immersive as a creative artist, brings us back to that quality of early childhood experience. For our visitors, who may be visiting an immersive experience for the first time, I want to keep the freshness and the immediacy of this."

\subsection{Individual artist responses}

Chen Mei-Tsen experiences time, space and memory as an urban nomad, observes Nature and studies the mutual relationships between living creatures and their biological, social, and environmental surroundings.

Fion Gunn contemplates the impact on her own work from curating other artists in large-scale exhibitions, creative journeys experienced through migration, displacement and transformation.

Maureen Kendal offers a vision of migration, trauma and joy. Experiencing an immersive world may open up deep wells of forgotten emotions, because we are all people, migrating from trauma, trying to survive in unfamiliar territories and seeking out connections.

Cleon Grant develops 3D structural, coherent and elegant virtual worlds. Using sound and every day scenarios to explore and experience the thoughts and feelings of someone before and after an anxious interaction.

Terri M Broughton: The basis of the sea's food chain is largely invisible. Tiny plants and plankton capture the sun's energy and are then converted into nutrients for the next chain of sea creatures, which is continuously passed on throughout the sea.

Nazia Parvez: The A-Maze group created a shared space in which I was able to connect with other artists. It provided a forum for us to discuss our experiences of the pandemic and the impact of lockdowns both collectively and individually. These discussions were a means to explore different ideas and themes.

Artists' statements are available online at: http://www.worldsinflux.com/artists-pages.html

\subsection{Mutual interview extracts}

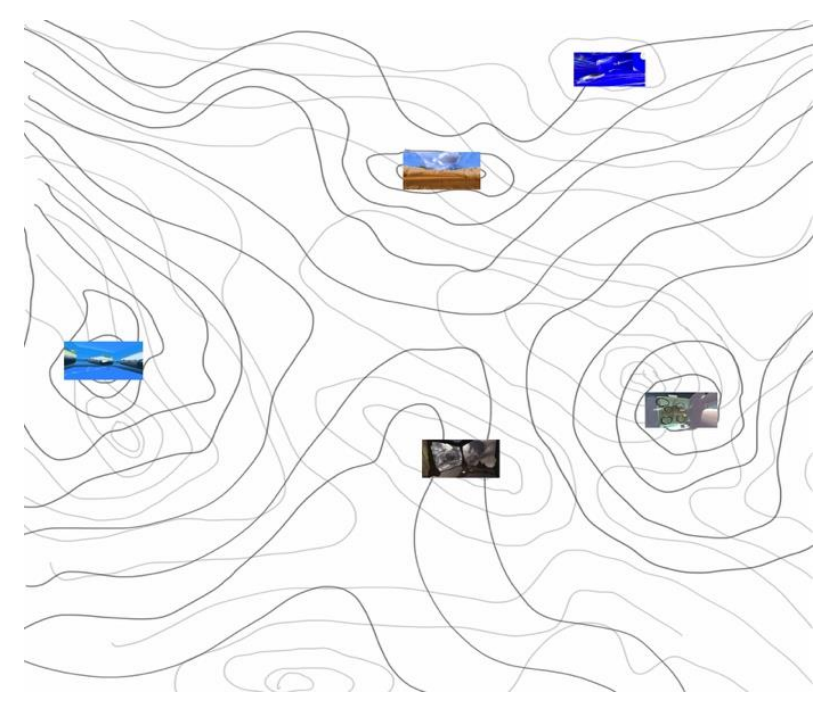

Figure 3: Visual evidence.

\section{Mei-Tsen}

"As artists we generally work on our own, we have our idea, we try to develop it ourselves and then create the work. It is a solitary process. Boundless has opened up another horizon for me, working with different artists of different ages from different cultures with different points of view, perceptions and personal lives. We've been questioning each other and it is interesting to learn about other artists' processes, because process is often the most fascinating aspect of creation."

"For me the aesthetic of displacement is like cartography - a physical, neurological and psychological map. Within this map we travel as though through a web from any point on any line to another. It's a kind of visual flux, these are weblike maps, not linear and they imply 3 dimensionality. The viewer can travel within the painting, investigating the work like a journey through a labyrinth."

"I use metaphor and analogy to describe what I am doing and also how we perceive mythological narratives and imagery. This allows us ways of inhabiting other life forms in our imaginations where for example I could be a jellyfish, a tree. We imagine another way of being in order to develop new perceptions and perspectives on our place in the universe."

Fion

"Even though the technology is different I am using a similar methodology in my work using Tilt Brush and the other digital technologies because I stitch things together. My VR creations include 3D models I've imported, some of these l've made 
myself and some have been made by other people, then I add to them, I transform them, I do things to them. Equally, the way I use the brushes, a lot of people use them as a painterly medium and I tend to use them more as a sculptural medium."

"The theatrical and dynamic potential is crucial to the telling and weaving together of the multiple stories: historical, global dimension and personal stories. In terms of how this collaboration works, my curatorial interests are evident: finding connections and pathways between the works of very different artists 'worlds."

"Curating in this way is like making a 4 dimensional quilt because of the element of time and travel which run through our project and, immersive technology brings in sound, movement, lighting, staging, all the other media as well."

Working with Virtual Tools and Immersive spaces: Maureen's questions to Fion

Maureen - what is different about the Immersive platform?

Fion - in my current work -1 am creating multimedia collage, stitching together - seemingly unrelated things except that these are connected when we view the nature of our world, our existence, our universe as being interconnected. The rationale is not linear and it works in a 4 dimensional way. What I love about immersive technology is that I can work in time as well. The passage of time is not just implied or encapsulated as it is in my 2/3D work, it is actual. Combining technologies and finding workaround solutions is an immensely engaging, if challenging process. When I work in this way using 3D artefacts I create myself, importing those created by others which I can then remix and alter and expand on, I am making a truly dynamic and inclusive collage. Creating in the virtual space, I am working with time and movement also and this is very much part of the 'theatrical' aspect of my practice and it is closely linked to my curatorial approach. So for example, I have created a complex densely forested space where I can explore and navigate different pathways and even get lost in it.

Chen Mei-Tsen, Fion Gunn, Maureen Kendal, Cleon Grant, Terri M Broughton, Nazia Parvez. Extracts from mutual interviews are available online at:

http://www.worldsinflux.com/artists-interviews.html

\section{REACHING OUT TO NEW AUDIENCES}

For the long-term future, it is envisaged that themes and featured artists can change and that other similar 'Hubs' can be created for different institutional partners and communities.

\subsection{Outputs:}

1. Filming the VR experience through the eyes of an avatar.

2. Structuring the search and user journeys through quizzes.

3. Blended delivery both physical, augmented, virtual and online.

4. Enabling user uploads from site-specific outreach sites and display outlets.

\subsection{Outcomes:}

1. Creation of new audience for the Immersive.

2. Evolution of the artists' practices.

3. Activism and transformational impact.

4. Trans-disciplinary global connectivity.

Currently A-Maze artists are identifying opportunities, investors and funds to develop the Minimum Viable Product (MVP) and scale up for a range of local, national and global partners. Opportunities for site-specific artist-technology projects have been identified. These opportunities seek transformative social/technical interventions, bridging historical, archaeological and cultural heritage, emerging community co-creation, converging with initiatives, which prioritise social, environmental and ecological well-being. "The gap between the "haves" and "have-nots" will widen further if technology access and ability remain disparate", (WEF 2021). Virtual Immersive Interactive Art facilitates participation by wider audiences, increases dialogical interventions between individuals and organisations, which benefit communities. This follows on in the tradition of artists' interventions with and on behalf of communities (Grant, 2004).

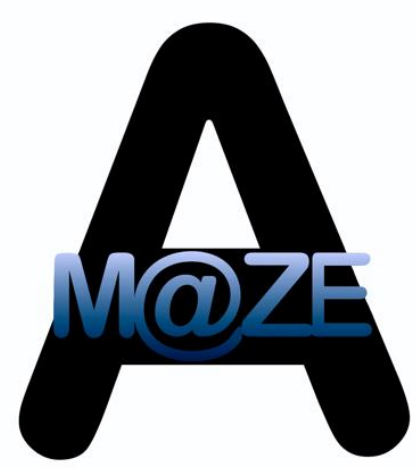

Figure 3: Visual evidence. 


\section{REFERENCES}

Arts Council National Lottery Project Grants, (2021). https://www.artscouncil.org.uk/projectgrants (retrieved 14th March 2021).

Boundless - Worlds In Flux. http://www.worldsinflux.com/ Calcagno, R. and Goy, J. (2014). 'Méduses-À la conquête des océans'. Institut Océanographique de Monaco / Éditions du Rocher.

Chen, M. Kendal, M. and the AMaze group, (2021) online discussion on navigational development.

Croally, Neil. (2007). Hecabe: Euripidean Polemic: The Trojan Women and the Function of Tragedy. Cambridge University Press. ISBN 0-521-04112-0.

Cybercare Ltd, Cybercare Trust. www.cybercare.org.uk / www.cybercare.support / www.cybercare.services. (retrieved 14th March 2021).

Demsar J., Hemelrijk C.K., Hildenbrandt H. and Bajec I.L. (2015). "Simulating predator attacks on schools: Evolving composite tactics" (PDF). Ecological Modelling. 304: 2233. doi:10.1016/j.ecolmodel.2015.02.018 (retrieved 14th March 2021).

Ducousso, P. (2019) 'Les méduses, une armada qui menace les océans'. Le Point Sciences https://www.lepoint.fr/sciences-nature/les-meduses-unearmada-qui-menace-les-oceans-17-07-2019-

2325202_1924.php (retrieved 14th March 2021).

Georgia Aquarium, Atlanta, US, https://www.georgiaaquarium.org/ (retrieved 14th March 2021).

Google (2021) Tilt Brush software. https://www.tiltbrush.com

Gottleib Zornberg, A. (2009) The Murmuring Deep: reflections on the biblical unconscious (book).

Gottleib Zornberg, A. "I am a Stranger:" Becoming Ruth (online video)

https://www.youtube.com/watch?v=sqg_apYelZk (retrieved 14th March 2021).

Grant, C. and the AMaze group (2021) online discussion on navigational implementation.

Grant, H.K. (2004) Conversation Pieces: Community and Communication in Modern Art, University of California Press.

Gunn, F. (2019) Odyssey: Explorations. Interactive exhibition with workshops and partnerships. https://www.tate.org.uk/whats-on/tate-liverpool/tateexchange/workshop/odyssey-explorations accessed 14/3/2021).

Gunn, F. (2019) Odyssey Explorations - the digital journey. https://www.odysseyexplorations.com/ (accessed 14/03/2021)

Gunn, F., Kendal, M., Mulla, M. (2020) An Exploration of How Artists Use Immersive Technologies to Promote Inclusivity. In: Weinel, J., Bowen, J.P., Diprose, G., and Lambert, N. (eds), EVA London 2020 (Electronic Visualisation and the Arts) 2020.

Gershwin, L., (2017) 'Méduses \& autres organismes gélatineux'. Éditions - Ulmer.

Gunn, F. (2020) BOUNDLESS - WORLDS IN FLUX (TEST VIDEO) https://youtu.be/Qk5SodovFCs (retrieved 14th March 2021).
Gunn, F. (2021) Kendal, M. and the Amaze group in conversation to develop this project.

Heraclitus (circa 535 BC/ BCE) Greek Philosopher. https://www.britannica.com/biography/Heraclitus, (retrieve d 14th March 2021).

Hudson. A. and Kendal, M. (2011) On the Beach - A handbook for using 3D virtual digital platforms like Second Life - 'the WEISL' - 'Writing Explorations in Second Life', Electronic Visualisation and the Arts (EVA 2011), UK, BCS, London.

Hudson. A. and Kendal, M. (2011) Second Life Handbook - WEISL - Writing Explorations In Second Life. SWAP funded publication (Supporting Writing for Assessment), London Metropolitan University.

Kendal, M. (2018), 'Conquer the Web' published by Legend.

Kendal, M., Mulla, M.,Thomazi, Freitas, E. (2019) How Artists Can Develop Their Artwork, Its Market and Deliver Their Creative Content for Virtual Reality Environments? In: Weinel, J., Bowen, J.P., Diprose, G., and Lambert, N. (eds), EVA London 2019 (Electronic Visualisation and the Arts) 2019.

Kendal, M. (2019) 'Cyber and You', published by Legend.

Institut Océanographique, Monaco https://www.oceano.org/ (retrieved 14th March 2021).

Le Brun, D. (2018), 'La Méduse-Les dessous d'un naufrage'. Éditions - Omnibus.

Le Prisme-Le magazine des urbains nomades, meitsen-chen-figure-de-l-artiste-nomade https://www.leprismemagazine.com/singlepost/2016/04/30/meitsen-chen-figure-de-l-artiste-nomade (retrieved March 2021).

Matisse, H.(1908) "Notes from a Painter," La Grande Revue, LII, 24, 25, December 1908, pp.481-485. (Translated in Matisse on Art, editor Jack D. Flam, E.P. Dutton, 1973)".

Médusarium@ Aquarium de Paris, France (accessed 2021) https://www.aquariumdeparis.com/medusarium/

Natural Selections, Trouvé Magazine - accessed (2021). http://www.trouvemag.com/journal/natural-selections

Qlone App (retrieved 2019) https://www.qlone.pro/

Radakov DV (1973) Schooling in the ecology of fish. Israel Program for Scientific Translation, translated by Mill H. Halsted Press, New York. ISBN 978-0-7065-13516 (accessed 14/3/2021).

Rodrigo, S. (2021) https://virtualrealityartist.co.uk/, (accessed 14/03/2021).

Stewart, S. (1993) (2007) On Longing, Narratives of the Miniature, The Gigantic, the Souvenir, the Collection. Published by Duke.

Unity Technologies (2021)

https://unity.com/solutions/game (retrieved 13th March 2021).

World Economic Forum (2021) The Global Risks report 2021

http://www3.weforum.org/docs/WEF The Global Risks Report_2021.pdf (retrieved 13th March 2021). 